\title{
A silent variable-count light-pulse gate
}

\section{FRANK R. WILKINSON \\ University of Western Ontario \\ London, Ontario, Canada}

A silent, variable-count light-pulse gate system is described for achieving low noise and controlled pulse train length.

There are occasions when a researcher wishes to deliver to the eye a series of light pulses with minimum rise and fall times and to have independent control over pulse duration, interpulse interval, spectral characteristics, and intensity. One solution is to use some form of episcotister, sectored disk, or shutter which periodically interrupts a light beam at a focal point. Such a system allows independent manipulation of spectral characteristics through the use of continuously operating sources and selective filters; intensity is controlled with neutral-density wedges or filters or by use of the inverse-square law. Since the source is operating continuously, incandescent sources can be used with their broad spectral and high energy output characteristics; the desired minimal pulse rise and fall times can still be achieved if the optics of the system are attended to.

Interrupted-beam systems, however, can pose problems in at least two areas: (1) noise, and (2) control of the number of light pulses in the train. This report describes one system for achieving low noise and controlled pulse train length. While not the only way this can be done, the system is flexible, easily adjustable, and can be built at relatively low cost out of readily available components without requiring a high level of electronics background. Figure 1 is a functional diagram of the system as a whole. Following some general remarks on various components of the system, we will deal in more detail with the gate unit and the signal source.

The basic system consists of a light beam interrupted by a small opaque flag or stop mounted on the shaft of a galvanometer or pen motor driven by a square-wave signal input. There is nothing new in this approach. The author saw such a device operating in the laboratory of S. H. Bartley at Michigan State University a few years ago, where the control signal was taken from a square-wave generator to produce a continuous train of pulses of light with a light-dark ratio of 1.0 and a frequency of approximately $30 \mathrm{~Hz}$. The purpose of the instrument was to demonstrate certain color changes that can occur in flickering lights. A noticeable feature was the silent operation coupled with precision control of frequency without the need for awkward timing devices such as stroboscopes or tachometers. The system described here is an extension of that device with the addition of a gate unit for producing trains of pulses of controlled length.
The pen motors used in Bartley's laboratory had been salvaged from old EEG machines, but such motors can also be secured in convenient form from Machines for Electronics, Inc., of Wilmington, Massachusetts, together with the necessary driver-amplifier circuitry. These are the R4 series pen motors and amplifiers. They are furnished with a power supply and blue ribbon connector that can be conveniently packaged in a $1455 \mathrm{G}$ Hammond cabinet. Once assembled, these units require only a signal input to drive the pen motor. The generation and control of this signal are the business of the signal source unit and the gate unit, respectively.

The gate unit is designed to receive a continuous train of electrical pulses as input, simultaneously squaring and counting the pulses and passing them to the driver amplifier (see Fig. 2). Counting and enabling of the gate output begins with a manually operated contact closure (S1 in Fig. 2). When the pulse count reaches a number between 1 and 127 inclusive (previously entered into a bank of binary switches, S3.9 in Fig. 2), the gate inhibits the passage of any further pulses until triggered again by the manual contact closure. Reset of the counter is automatically achieved by the first input pulse following the termination of counting. Regardless of when the contact closure occurs, enabling of the output does not take place until the onset of the next succeeding positive-going pulse edge, at which point counting also begins. An additional switch (S2 in Fig. 2) allows the counting operation to be disabled so that upon contact closure, the gate will continually pass signal pulses until manually stopped (reset S2 with S3-9 all in 0 position).

The switching and counting are done with integrated circuits. Figure 2 shows the schematic.

According to the IC specifications, signal inputs to the gate unit must be essentially square for the counter to count accurately. The rise and fall time requirements are specified as $\leqslant 10 \mathrm{nsec}$. In order, therefore, to avoid placing too stringent a restriction on the signal source, the input signal is shaped by passing it through a SN7413N Schmitt trigger. The low state of the signal input to the Schmitt trigger should be $<0.6 \mathrm{~V}$, while the high state should be $>2.0 \mathrm{~V}$. Maximum operating

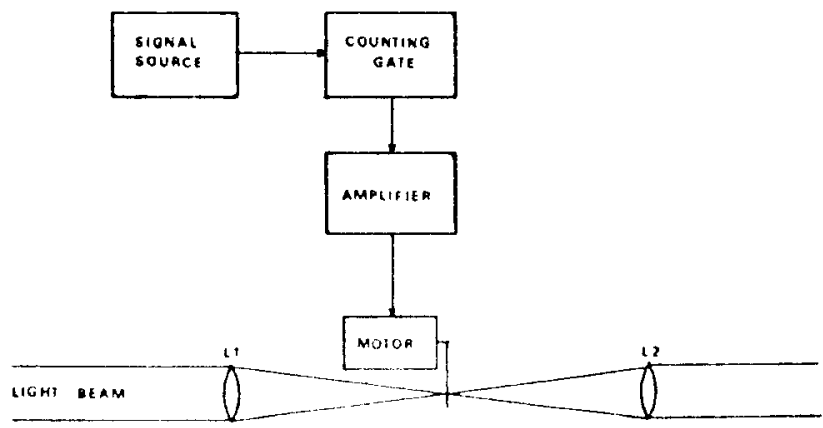

Fig. 1. Functional diagram of the light-pulse gate system. 


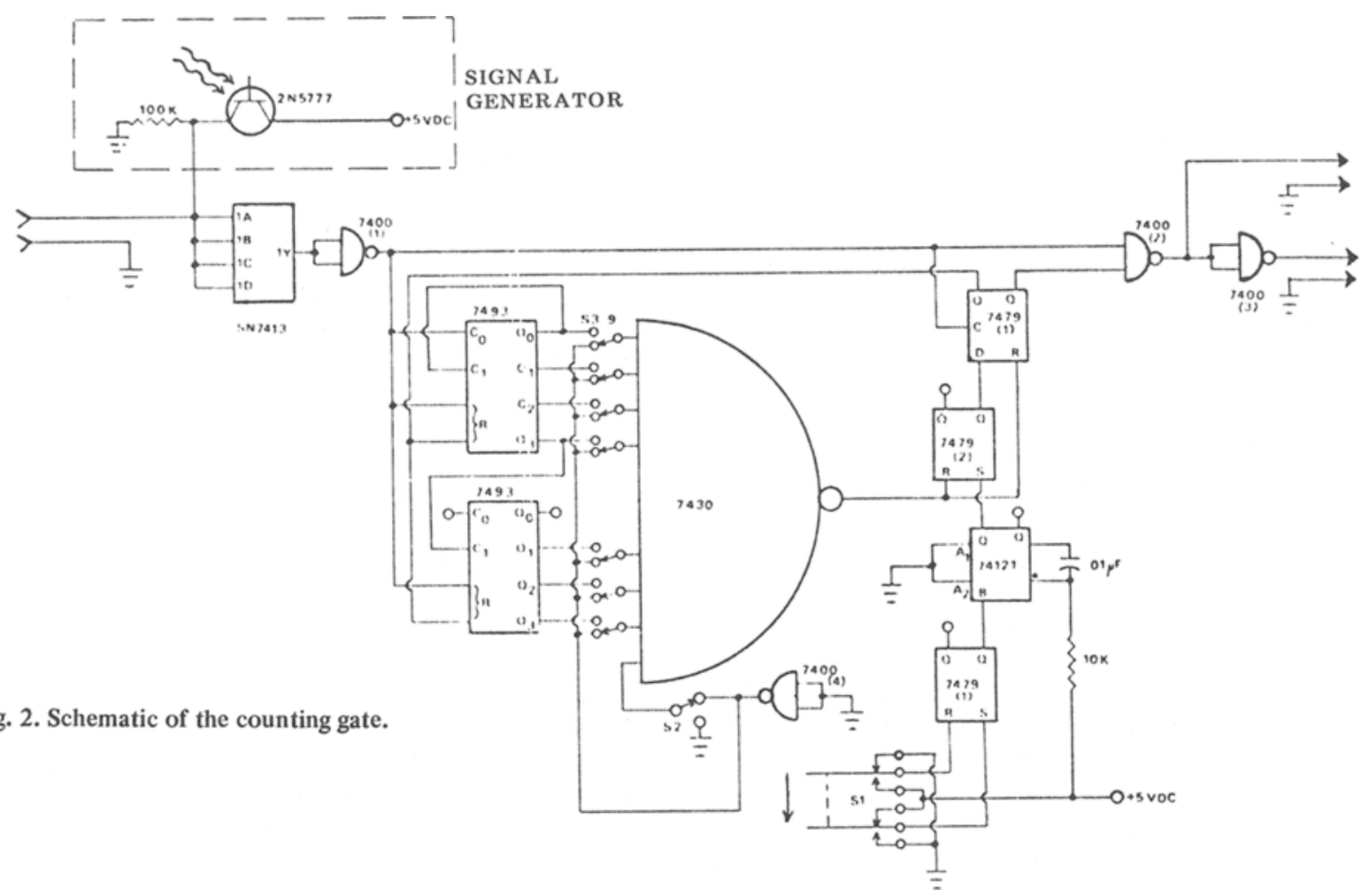

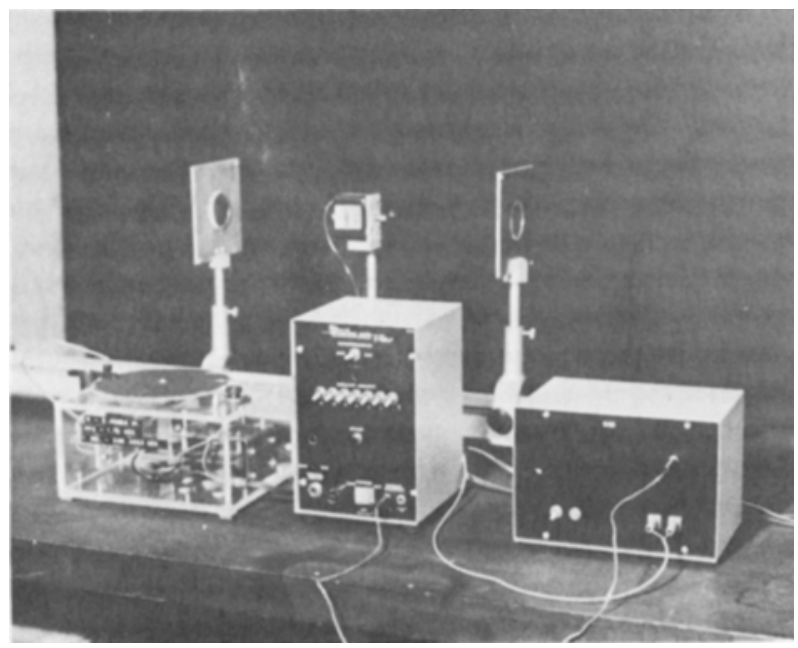

Fig. 3. The variable-count light-pulse gate, prototype model.

frequency of the counter is $>10 \mathrm{MHz}$. The gate can thus handle any frequency to which the pen motor can respond.

The problem that remains is to generate trains of pulses of the desired frequencies and spacing. The solution employed here consists of a constant-speed sectored disk with the pattern of the sections corresponding to the time sequence characteristics of the desired signal. Figure 3 shows the complete system as we have packaged it. The disk rotates horizontally, riding on a transparent plastic turntable over a small light source and in the plane of a focal point. The alternate exposure and blockage of the light is detected by a simple circuit, the sensor of which is a 2N5777 light-sensitive transistor (see Fig. 2, signal generator). In our configuration, all components of this sensing circuit except the transistor are packaged with the gate assembly. The transistor is on the end of a $6-\mathrm{ft}$ two-conductor line poised over the rotating disk. The disk is driven by a synchronous timing motor with a fixed number of revolutions/minute. The disk is slipped on and off the turntable like a phonograph record and can easily be changed in a few seconds. Power to the transistor circuit is supplied from the gate unit power supply.

Other means of generating the necessary input signals are possible, e.g., trains of square pulses recorded on and read from magnetic tape loops, mechanically driven switch closures (if switch bounce is adequately controlled) and square-wave signal generators, including sophisticated (and expensive) models with continuously and independently variable pulse- and interpulse-duration controls. Whatever the source, the gate unit will shape and pass sequences of pulses, on command, up to some preset number or continuously. Furthermore, two or more pen motors can be run simultaneously, using the normal and inverted gate outputs to provide the effect of switching from one light channel to another and back again. In this configuration, the pen motors will operate $180 \mathrm{deg}$ out of phase, with the gating operations being virtually simultaneous. Thus, the gate would have utility in a variety of experimental paradigms, including certain tachistoscopic applications. The use of two such gate units would allow overlapping pulses. The signals for such pulses could be derived from separate sensors positioned over a single rotating disk.

(Received for publication January 9, 1973; revision received Marclı 27, 1973.) 